\title{
Ionization Profile Monitor for SNS
}

BNL/SNS TECHNICAL NOTE

NO. 059

R. Connolly, P. Cameron, T. J. Shea and R. Witkover

March 1, 1999

ALTERNATING GRADIENT SYNCHROTRON DEPARTMENT BROOKHAVEN NATIONAL LABORATORY UPTON, NEW YORK 11973 



\title{
IONIZATION PROFILE MONITOR FOR SNS
}

\author{
R. Connolly, P. Cameron, T.J. Shea, and R. Witkover \\ Brookhaven National Lab, Upton, New York 11973
}

\section{INTRODUCTION}

The Spallation Neutron Source (SNS) consists of a $1 \mathrm{GeV}$ linear accelerator (linac), a storage ring, a target, and connecting beam-transport lines [1]. The linac delivers a 1.04-ms-long macropulse which is chopped into about $10^{3}$ pulses of $10^{11}$ protons each. As this pulse enters the storage ring from the linac each chopped pulse lines up longitudinally with all proceeding pulses forming a single bunch about 550ns long and a 250-ns gap. During the accumulation process the transverse profiles of the beam will be measured with ionization profile monitors (IPM's).

An IPM measures the distribution of electrons freed by ionizing collisions of the beam with residual gas in the beamline [2,3]. The electrons are swept from the beamline by a transverse electric field, amplified by a microchannel plate (MCP), and collected by a circuit board with strip anodes oriented parallel to the beam axis. A uniform magnetic field, parallel to the sweep electric field, counters the defocusing effects of space charge and recoil momentum [4]. During each bunch passage the charge pulses are integrated, amplified, and digitized for display as a profile histogram.

The purpose of this paper is to evaluate the performance requirements the SNS will demand of an IPM and to propose a development path to meet these requirements. In the following sections we discuss electron focusing, proposed detector design, anticipated signal sizes, and requirements for the electronics. It is logical at this time to propose that the SNS IPM will be similar to those used in RHIC. However only a prototype has been tested for RHIC. Beam tests of the RHIC IPM will begin in the summer of 1999 and these tests will provide valuable information for the design of IPM's for SNS.

All model calculations assume a full beam emittance of $120 \pi \mathrm{mm} * \mathrm{mrad}$ and $\beta=10 \mathrm{~m}$ giving a beam $5 \sigma$ radius of $35 \mathrm{~mm}$. Since the chopped linac pulses are injected around the circumference of a ring in transverse phase space, the transverse profiles of the accumulated bunch in the ring are approximately flat with peaks at both ends [5]. The calculations model the incoming chopped pulses with Gaussian transverse profiles and the full beam with a rectangular transverse profile.

\section{ELECTRON KINEMATICS}

The density of ionization events in the residual gas is a map of the beam distribution. An IPM sweeps the electrons formed in these ionizing collisions from the beamline with a transverse electric field and measures their distribution. While the electrons are being swept from the beampipe they can move parallel, but not perpendicular, to the collectors anodes. However, the ionizing event imparts momentum to each electron, and the electron is released in the space-charge electric field of the beam. The impulse from the collision and the transverse component of the space-charge electric field can move the electron perpendicular to the collector channels resulting in a measured profile which is different than the beam's. To counter these effects the detector is placed in a magnetic field oriented parallel to the applied electric field.

The space-charge electric field of the beam bunch is a radial field whose strength increases with radius to a maximum near the beam's edge. Each electron is released in an electric field with a component parallel to the applied sweep field (which causes no error) and a component perpendicular to it. Without magnetic confinement the perpendicular component, $\mathrm{E}_{\perp}$, accelerates the electron across collector channels. With the magnetic field the electron is in perpendicular electric and magnetic fields, and it will drift with a cycloidal motion in the direction defined by the vector $\mathbf{E} \times \mathbf{B}$ (parallel to the collector anodes) with a velocity of,

$$
\mathbf{v}=(\mathbf{E} \times \mathbf{B}) / \mathrm{B}^{2}
$$


and a gyration radius of,

$$
\mathrm{R}=\mathrm{Em} / \mathrm{qB}^{2}
$$

A bunch containing $2 \times 10^{14}$ protons within a radius of $35 \mathrm{~mm}(\beta=10 \mathrm{~m})$ will have a maximum space-charge field of about $10^{5} \mathrm{~V} / \mathrm{m}$. In a magnetic field of $0.1 \mathrm{~T}$, an electron in the maximum transverse field will travel parallel to the collector channels at $10^{6} \mathrm{~m} / \mathrm{s}$ with a gyration radius of $<70 \mu \mathrm{m}$. Profile broadening by the space-charge field is insignificant. By the same mechanism transverse components in the sweep electric field will not cause measurement errors. A sweep field of $150 \mathrm{kV} / \mathrm{m}$ will remove electrons is under $10 \mathrm{~ns}$ so the maximum longitudinal drift is less than $1.0 \mathrm{~cm}$.

The second defocusing effect is the momentum impulse from the ionizing collision. The energy spectrum of recoil electrons extends to $3.0 \mathrm{MeV}$ in SNS but over 95\% of them have energies <500 eV [6]. In a magnetic field the electron trajectory is a helix whose axis is parallel to the magnetic field and whose radius varies linearly with the transverse component of momentum. A $0.1 \mathrm{~T}$ field confines a $500 \mathrm{eV}$ electron to a Larmor radius of $<0.8 \mathrm{~mm}$ which is about $2 \%$ of the beam radius. By placing the detector in a field of $0.1 \mathrm{~T}$ most of the electrons are collected on the anodes over which they are formed.

\section{DETECTOR DESIGN}

Figure 1 shows the RHIC IPM. The detector, three electrical feedthroughs, and a quartz window (not shown) are mounted on a single 10" conflat flange. This flange is mounted on a side port of the detector vacuum chamber made from $100 \times 150 \mathrm{~mm}$ stainless steel tubing. The vacuum chamber is mounted in the gap of a ' $\mathrm{C}$ ' dipole permanent magnet.

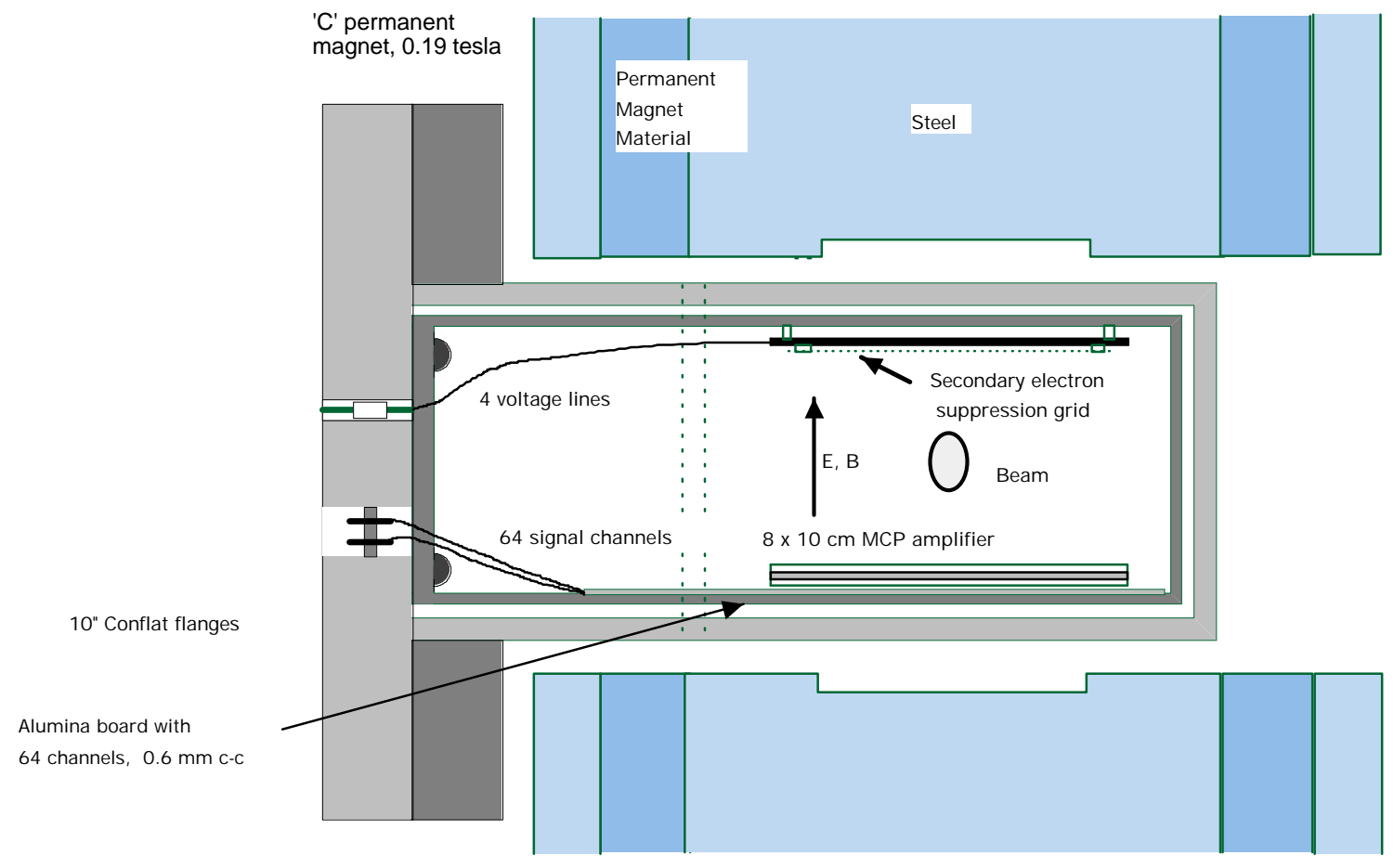

Figure 1. Cross section of RHIC IPM. View is looking down the beamline. 
Two rectangular brackets hold the collector board and a double-plate MCP amplifier on one side of the beam, and a sweep-field electrode and secondary electron suppression grid on the other side. The collector board is $0.625 \mathrm{~mm}$-thick alumina, plated with gold, and etched with 64 collection anodes. At one end of each anode a plated through hole conducts the charge to a trace on the back which takes the signal to the edge of the board. A wire connects each trace to a pin on a vacuum feedthrough.

For RHIC we use permanent-magnet dipoles producing fields of 0.19T. Data taken with the RHIC prototype IPM indicate that $0.1 \mathrm{~T}$ is adequate for accurate imaging. However the magnets were built with strontium-ferrite bricks (1"x4"x6") and integral multiples of 14 were required for field uniformity. A 14-brick magnet was too weak so we used 28 bricks. In addition to the detector magnet, a second magnet is mounted $0.5 \mathrm{~m}$ from the first with its field oriented opposite to the first. The beam experiences a slight transverse offset passing through the chamber but emerges with the direction of its trajectory unchanged. For SNS we will use three magnets for each IPM with the two end magnets causing half of the beam deflection as the center IPM magnet.

The SNS beam has a full-beam $(5 \sigma)$ emittance of $120 \pi \mathrm{mm} * \mathrm{mrad}$ so the design beam diameter at $\beta=10 \mathrm{~m}$ is $35 \mathrm{~mm}$. Collimators in the beamline restrict the full-beam emittance to $180 \pi \mathrm{mm} * \mathrm{mrad}$ so the maximum beam size where $\beta=10 \mathrm{~m}$ is $84 \mathrm{~mm}$ diameter. Using these beam sizes a detector with 64 collector channels spaced $1.4 \mathrm{~mm}$ apart covering a diameter of $90 \mathrm{~mm}$ would be appropriate. The full beam would produce signals in 50 channels and all beam, including halo, would cover 60 channels. A clear aperture of twice the maximum beam diameter will be used to minimize background from scattered beam.

\section{SIGNAL SIZE}

It is expected that the residual gas in SNS will be about two thirds $\mathrm{H}_{2}$ and one third $\mathrm{CO}$ [7]. The ionization rate produced by a proton traveling through a gas decreases with increasing energy to a minimum at about $1 \mathrm{GeV}$ [8]. The ionization rate consists of primary ionization rates and secondary events caused by the energetic recoil electrons. Figure 2, copied from ref. 8, shows the approximate relationship between primary ionization rates and atomic number for gases at atmospheric pressure for a minimum ionizing particle.

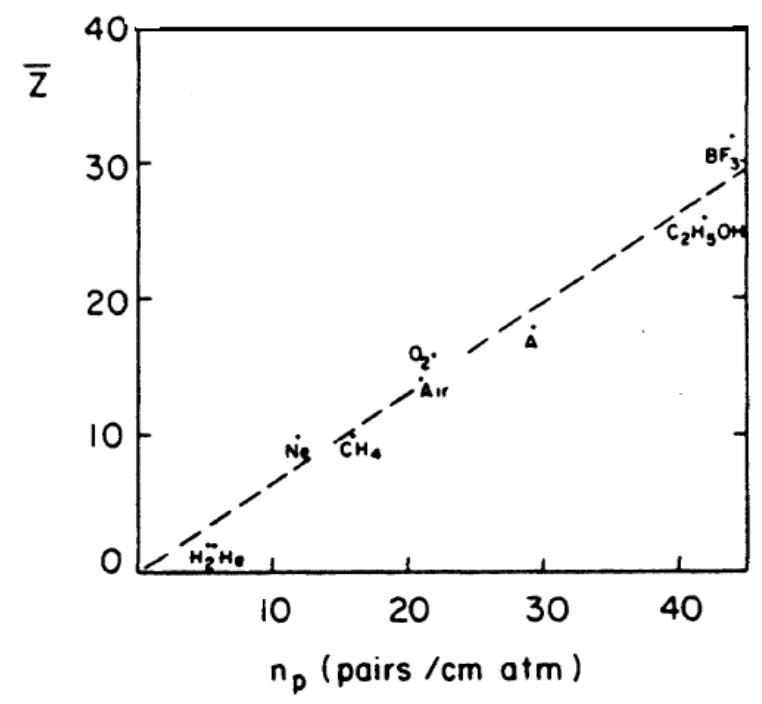


Figure 2. The number of primary ionizing events produced by $1 \mathrm{GeV}$ protons per $\mathrm{cm}$ at one atmosphere pressure plotted vs. the atomic number of the gas. This plot is copied from ref. 9.

The measured primary ionization rate in $\mathrm{H}_{2}$ is 5.2 ion pairs/cm and, from this graph, we estimate the ionization rate in $\mathrm{CO}$ to be $22 \mathrm{i} . \mathrm{p} . / \mathrm{cm}$. Therefore a $1 \mathrm{GeV}$ proton in this mixture of gases at one atmosphere pressure produces 10.7 i.p./cm. Each bunch passage past the detector will create $\mathrm{N}$ detected electrons where,

$\mathrm{N}=[1.4 \times 10-2][$ Pressure(torr)][Number of protons][Detector length $(\mathrm{cm})][$ Detection efficiency].

An MCP detection efficiency is about $36 \%$ for electrons with energies of a few keV. Galileo produces rectangular MCP with active areas of $74 \times 93 \mathrm{~mm}$ [9]. A simple detector configuration would be to cover the $900 \mathrm{~mm}$-wide collector with a single MCP giving a detector length of $74 \mathrm{~mm}$. The number of detected electrons then is,

$$
\mathrm{N}=\left[3.7 \times 10^{-2}\right][\text { Pressure(torr) }][\text { Number of protons }]
$$

With a beamline pressure of $5 \times 10^{-9}$ torr an injected bunch at half the design intensity would produce 10 electrons and the full beam intensity of $2 \times 10^{14}$ protons would produce $3.7 \times 10^{4}$.

It is not feasible to build a system with this large of dynamic range. Primarily the IPM will be used to measure the profile toward the end of the machine cycle. The MCP will remain biased and the sweep field will be switched on and off to record the interesting portion of the machine cycle. For machine studies of the injection process the chamber pressure will be raised by a controlled leak with feedback. The design injected beam bunch is $10^{11}$ protons with an emittance of $0.14 \pi \mathrm{mm} * \mathrm{mrad}$. If the IPM is located at $\beta=10 \mathrm{~m}$ the rms width of the beam is $1.2 \mathrm{~mm}$. This will deliver signal to only 3-4 IPM channels. This will not provide an emittance measurement but will allow monitoring of phase-space filling.

A smoke ring distribution in phase space results in a profile which is approximately rectangular. With $3.7 \times 10^{4}$ electrons covering 50 channels each channel receives about 740 . If a single MCP is operated with a gain of $10^{4}$, then each channel would deliver a current of about $2 \mu \mathrm{A}$ during the beam pulse. These currents will be brought out of the tunnel on coaxial cable and amplified and digitized.

\section{ELECTRONICS}

\section{Microchannel plate}

If a MCP is used as the primary amplifier and the profile of every bunch is to measured, the maximum detector output charge per beam bunch is limited by the MCP strip current. When a MCP is used as a dc current amplifier, the gain is constant until the output current exceeds approximately $10 \%$ of the strip current [10]. A $74 \times 93 \mathrm{~mm}$ extended dynamic range MCP has a strip current of 325-1500 $\mu \mathrm{A}$ when biased at $1 \mathrm{kV}$. If we assume a current of $1.0 \mathrm{~mA}$ per plate this gives an upper limit on the signal current density of $0.014 \mu \mathrm{A} / \mathrm{mm}^{2}$. Each channel is $104 \mathrm{~mm}^{2}$ so the output current per channel should kept below $1.5 \mu \mathrm{A}$. With a bunch frequency of $1.19 \mathrm{MHz}$ this gives a maximum charge per pulse per channel of $1.3 \mathrm{pC}$. The example worked out in the previous section gave $1.2 \mathrm{pC} / \mathrm{bunch}$.

A MCP has a finite lifetime caused by electron scrubbing of the channel walls near the plate output. The gain gradually drops, falling to about $90 \%$ of new after the plate has delivered about $0.1 \mathrm{C} / \mathrm{cm}^{2}$. Each collector channel has an area of about $1 \mathrm{~cm}^{2}$ so the lifetime of the plate will be approximately $8 \times 10^{10}$ measured bunches. If 100 turns are measured each machine cycle the plate change interval will be $8 \times 10^{8}$ cycles. Also the finite lifetime requires the plate bias voltage be interlocked to the measurement system so it is only on during measurements. 


\section{Charge integration}

To keep the MCP from saturating during the machine cycle the charge per channel for each beam pulse has to be limited to about $1.0 \mathrm{pC}$. For a reasonable dynamic range across the profile the electronics have to be sensitive to integrated charges of $10 \mathrm{fC}$. Such low quantities of charge are difficult to measure with gated integrators. For instance the Burr-Brown ACF2101 gated integrator has an integration capacitor of $100 \mathrm{pF}(10 \mathrm{mV} / \mathrm{pC})$ and typical charge injection at reset and read of 100-200 fC [11]. There are ways to compensate for charge injection or to subtract it from the signal, but we do not know if it has been done well enough to achieve the required charge sensitivity. If single-turn profiles are not needed, a profile from many turns can be gathered in a single integration cycle thus reducing the charge-injection problem.

During the machine cycle the space charge electric field of the beam goes from zero to $>10^{5} \mathrm{~V} / \mathrm{m}$. The field from the proton beam will keep electrons in the beam pipe during bunch passage. If electrons are to be extracted during the bunch passage the sweep field has to be larger than the maximum spacecharge field. Since the maximum space charge field is not an exactly predictable quantity some overhead will have to be allowed. Probably an applied field of $150 \mathrm{kV} / \mathrm{m}$ would be adequate for all situations. This is achievable with $15 \mathrm{kV}$ applied over a $10 \mathrm{~cm}$ gap.

Another possible data-collection method is to amplify the signals and digitize them many times during the bunch passage. The VME digitizers developed for RHIC could digitize the profiles five times during one bunch passage. Integration over the beam pulse would be done by adding up the several profiles collected during one bunch passage. This is the proposal for the beam position monitor system [12].

\section{DISCUSSION}

The profile of the SNS beam can be measured with a detector similar to that used for RHIC. Two challenges are to integrate the very small charge signals over the 550ns bunch and to follow three decades of beam current. The charge integration might be done with either low-noise gated integrators or with fast digitizers. The large beam dynamic range will be handled by designing a system to measure beam profiles of the full beam and creating a local pressure bump with a small, controlled leak of an easily pumped heavy gas such as $\mathrm{CO}$ for machine studies earlier in the cycle.

Some care will be required when locating the detector. MCP's are sensitive to any radiation which produces secondary electrons. The detector cannot be near any beamline location where beam loss is likely such as scrapers or collimators. All of the beamline components of the detector have to lie outside of the maximum beam envelope defined by the collimator. With the intense beam of SNS it would be a good idea to place an annular shield in the beamline upstream from the IPM to keep beam-generated $\mathrm{x}$-rays from causing spurious signals.

One final concern is the operation of MCP's in a high radiation environment. Any radiation which produces secondary electrons can excite a channel. An MCP is sensitive to x-rays with wavelengths as short as $0.12 \AA$. It is possible that radiation will cause a large background with the channel plate biased. This has to be investigated.

\section{REFERENCES}

1. $\quad$ SNS Design Manual

2. W.H. DeLuca, "Beam Detection Using Residual Gas Ionization", IEEE Trans. Nucl. Sci., NS16, 813 (1969).

3. A.N. Stillman, et. al., "An Ultrahigh Vacuum Beam Profile Monitor”, Rev. Sci. Instrum., 63 (6) (1992)

4. R. Connolly, P. Cameron, W. Ryan, T.J. Shea, R. Sikora, and N. Tsoupas, “A Prototype 
Ionization Profile Monitor for RHIC", Proceedings of the 1997 Particle Accelerator Conference.

5. A.U. Luccio, D. Maletic and F.W. Jones, "Injection and RF Capture in the NSNS; A Numerical Simulation in 6 Dimensions," BNL/NSNS Technical Note No. 004, Brookhaven National Laboratory, Upton, NY.

6. J. D. Jackson, "Classical Electrodynamics," p. 430, John Wiley and Sons, New York, 1962.

7. Dick Hseuh, Private communication.

8. F. Sauli, "Principles of Operation of Multiwire Proportional and Drift Chambers," CERN 77-09 (1977).

9. Galileo Electro-Optics Corp., Sturbridge, MA 01566.

10. J. Wiza, "Microchannel Plate Detectors", Nucl. Instr. and Meth. 162 (1979) 587.

11. Burr-Brown Corp., Tucson, AZ 85734.

12. T.J. Shea, P.R. Cameron, and W.A. Ryan, "Design Study of Position Monitoring in the SNS Ring", Brookhaven National Laboratory, Upton, NY 11973. 